\title{
Erratum to: Semicontinuity of the solution set to a parametric generalized strong vector equilibrium problem
}

\author{
Q. L. Wang • Z. Lin • X. B. Li
}

Published online: 4 July 2014

(C) Springer Basel 2014

\section{Erratum to: Positivity DOI 10.1007/s11117-014-0273-9}

The original publication of the article contains an error which need to be amended as mentioned below:

In the original paper, we obtained an important lemma (i.e. Lemma 3.1).

Lemma 3.1 For any given $f \in C^{*} \backslash\left\{0_{Y^{*}}\right\}$. Suppose that the following conditions are satisfied:

(i) $A(\cdot)$ is continuous with nonempty compact values on $\Lambda$.

(ii) $F$ is u.s.c. with nonempty compact values on $B \times B \times \Lambda$.

(iii) For any $\mu \in \Lambda$,

$$
\left.\left(i n f_{z \in F(x, y, \mu)}\right) f(z)\right)\left(i n f_{z \in F(y, x, \mu)} f(z)\right) \leq 0, \quad \forall x, y \in A(\mu) .
$$

(iv) For any $\mu \in \Lambda$, assumption (A) holds for $f$.

Then, $S_{f}(\cdot)$ is l.s.c. on $\Lambda$.

The online version of the original article can be found under doi:10.1007/s11117-014-0273-9.

Q. L. Wang $(\varangle) \cdot$ Z. Lin $\cdot$ X. B. Li

College of Sciences, Chongqing Jiaotong University, Chongqing 400074, China

e-mail: wangq197@126.com

Z. Lin

e-mail: linzhi7525@163.com

X. B. Li

e-mail: xiaobinglicq@126.com 
In the proof of the lemma in the original paper, we need prove $y_{0}=x_{0}$ to obtain a contradiction. For the purpose, by applying (3) of the original paper and $\inf f_{z \in F\left(x_{0}, y_{0}, \mu_{0}\right)} f(z) \leq 0$, we obtained Page 6, line 14 of the original paper

$$
\inf f_{z \in F\left(y_{0}, x_{0}, \mu_{0}\right)} f(z)=0
$$

Then, by assumption (iv), we got that $y_{0}=x_{0}$.

In fact, (1) should be replaced by $i n f_{z \in F\left(x_{0}, y_{0}, \mu_{0}\right)} f(z)=0$. 